\title{
Magnetic bipolar transistor
}

\author{
Jaroslav Fabian ${ }^{\text {a) }}$ \\ Institute for Theoretical Physics, Karl-Franzens University, Universitätsplatz 5, 8010 Graz, Austria \\ Igor Žutićc) and S. Das Sarma \\ Condensed Matter Theory Center, Department of Physics, University of Maryland, \\ College Park, Maryland 20742
}

(Received 2 July 2003; accepted 10 November 2003)

A magnetic bipolar transistor is a bipolar junction transistor with one or more magnetic regions,
and/or with an externally injected nonequilibrium (source) spin. It is shown that electrical spin
injection through the transistor is possible in the forward active regime. It is predicted that the
current amplification of the transistor can be tuned by spin. (c) 2004 American Institute of Physics.

[DOI: $10.1063 / 1.1637954]$

We propose ${ }^{1}$ a novel device scheme-the magnetic bipolar transistor (MBT) — which builds on the existing technology (bipolar junction transistor ${ }^{2,3}$ ), adding spin degrees of freedom to the current carriers. A MBT is a bipolar spintronic device: its functionality is defined by the transport properties of electrons, holes, and their spins. While bipolar spintronics $^{4-7}$ still relies on experimentally demonstrated fundamental physics concepts (such as spin injection, ${ }^{8-11}$ spin filtering, ${ }^{12}$ or semiconductor ferromagnetism ${ }^{13-15}$ ) rather than on working devices, recent experiments ${ }^{16,17}$ on spin injection through bipolar tunnel junctions prove the potential of the spin-polarized bipolar transport for both fundamental physics and useful technological applications. Materials progress towards bipolar spintronic devices, including the MBT, ${ }^{1}$ is reviewed in Ref. 18 , while a prototype of GaAsbased MBT has recently been fabricated. ${ }^{19}$ Here we analyze MBTs (other types of spin transistors were proposed in Refs. 20-26), with a magnetic base and a source spin in the emitter. We predict that spin can accumulate in the collector due to the electrical spin injection, and that the current amplification of MBTs can be controlled by spin.

Crucial to MBTs is the use of magnetic semiconductors where the splitting of the carrier bands produces spinpolarized electrons or holes with the spin polarization of $10 \%$ or more. The carrier band splitting can be of the Zeeman or the exchange type. The former arises from large $g$ factors (for example, in $\mathrm{Cd}_{0.95} \mathrm{Mn}_{0.05} \mathrm{Se}$ the $g$ factor exceeds $500,{ }^{27}$ while it is as large as $50 \mathrm{in} \mathrm{InSb}$ at room temperature), and an application of a magnetic field, while the latter comes from the exchange coupling in ferromagnetic semiconductors (about $10 \mathrm{meV}$ ). In addition to the equilibrium spin, a nonequilibrium (source) spin can be generated in the emitter with external spin injection, electrical or optical. ${ }^{28}$

Our model is described in Fig. 1. We consider an $n p n$ structure doped with $N_{d e}$ donors in the emitter, $N_{a b}$ acceptors in the base, and $N_{d c}$ donors in the collectors. There are two depletion layers: one between the emitter and the base, the other between the base and the collector. The transistor is a three terminal device: there is a contact with an external

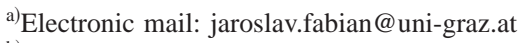

${ }^{b)}$ Electronic mail: igorz@physics.umd.edu
}

electrode at each region, generating bias $V_{b e}$ across the emitter-base and $V_{b c}$ across the base-collector depletion layer. The base is magnetic. For simplicity only electrons are spin polarized. The equilibrium spin polarization in the base is $\alpha_{0 b}=\tanh \left(q \zeta / k_{\mathrm{B}} T\right),{ }^{6}$ where $2 q \zeta$ is the conduction band spin splitting (assumed to be uniform across the base) and forward

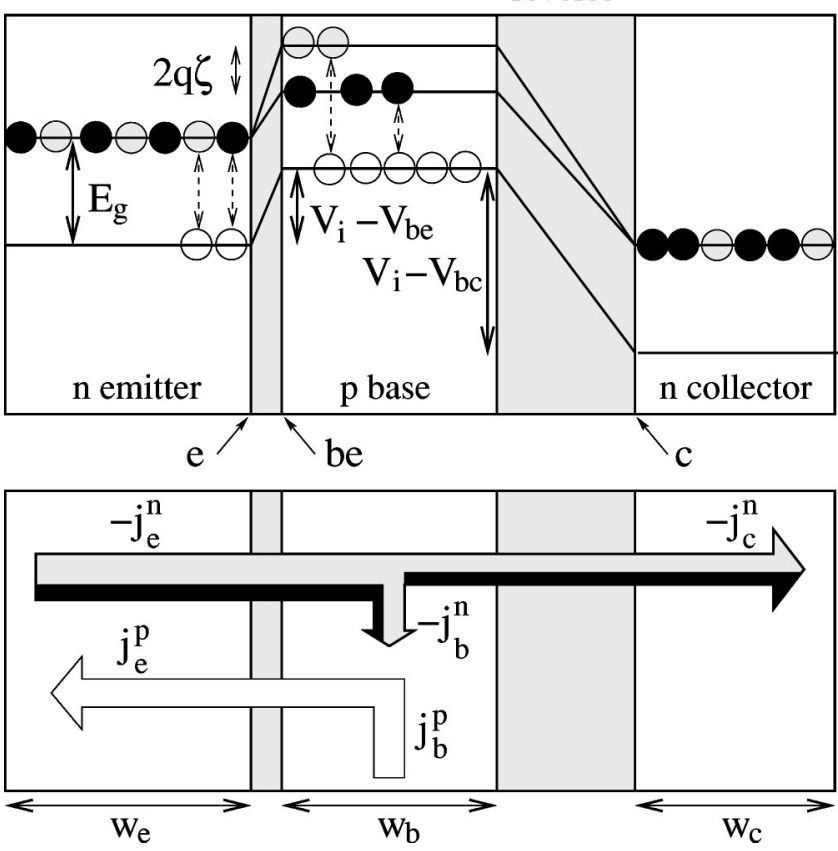

FIG. 1. The scheme of an $n p n$ transistor with a magnetic base. The top figure shows the bands. The conduction band is separated by the band gap $E_{g}$ from the valence band, and has a spin splitting (Zeeman or exchange) of $2 q \zeta$, leading to the equilibrium spin polarization $\alpha_{0 b}=\tanh \left(q \zeta / k_{\mathrm{B}} T\right)$, constant across the base. Holes are unpolarized. The spin is indicated by the shade of the circles (dark and light). The emitter-base junction is forward biased with voltage $V_{b e}>0$ lowering the built-in voltage $V_{i}$ and narrowing the depletion layer (shaded), while the base-collector junction is reverse biased with voltage $V_{b c}<0$, widening the depletion layer. Electrons flow easily from the emitter to the base, where some of them recombine (dashed lines) with holes, the rest being swept by the electric field in the basecollector depletion layer to the collector. Holes, which are the large part of the base current, flow to the emitter. The flow of electrons and holes is depicted ( $j$ are the corresponding charge currents) in the bottom figure, where also the effective widths $w$ are indicated. Symbols $e$, be, and $c$, which stand for the regions at the edges of the depletion layers, are used to label the corresponding electron and spin densities. The electron flow is spin-polarized, as indicated by the shading of the arrows. 
$k_{\mathrm{B}} T$ is the thermal energy. The nonequilibrium spin polarization injected externally into the emitter is $\alpha_{e}$. We assume that this source spin is injected within the spin diffusion length of the emitter-base depletion layer so that $\alpha_{e}$ is a representative value also in the $e$ region at the edge of the layer (see Fig. 1). The equilibrium number of electrons in the base depends on the equilibrium spin polarization, ${ }^{7}$

$$
n_{0 b}=\left(n_{i}^{2} / N_{a b}\right)\left(1 / \sqrt{1-\alpha_{0 b}^{2}}\right),
$$

where $n_{i}$ is the intrinsic carrier density. The equilibrium number of holes in the emitter is $p_{0 e}=n_{i}^{2} / N_{d e}$. For simplicity we assume that the electron and hole diffusivities $D_{n}$ and $D_{p}$, the electron and hole diffusion lengths $L_{n}$ and $L_{p}$, and the electron spin diffusion length $L_{s}$, are all uniform. The effective widths $w$ (which depend on the biases as well as on $\alpha_{0 b}{ }^{7}$ ) of the three bulk regions are defined in Fig. 1.

We consider the most useful forward active (also called amplification) regime of the transistor, where the emitterbase depletion layer is forward biased, $V_{b e}>0$, and the basecollector junction is reverse biased, $V_{b c}<0$, as shown in Fig. 1. Furthermore, we assume the small injection limit where the excess (injected) electron densities anywhere in the structure are smaller than the equilibrium densities determined by the doping. The resulting flow of electrons and holes is depicted in the bottom part of Fig. 1. Consider the electrons first. As the barrier between the emitter and the collector is lowered by $V_{b e}$, the electrons flow easily to the base, forming the electron emitter current $j_{e}^{n}$. In the base the excess electrons either recombine with holes, producing the base recombination current $j_{b}^{n}$, or diffuse towards the basecollector depletion layer. This layer is reverse biased so that all the electrons reaching it from the base are swept by the large electric field to the collector, forming the collector current $j_{c}^{n}$. Holes need to be supplied from the base to go in the forward direction to the emitter, forming the hole base, $j_{b}^{p}$, and the hole emitter, $j_{e}^{p}$, currents. The total emitter current is $j_{e}=j_{e}^{n}+j_{e}^{p}$ and the total collector current is $j_{c}=j_{c}^{n}$. The base current is $j_{b}=j_{e}-j_{c}$. The current amplification coefficient (gain) is defined as $\beta=j_{c} / j_{b}$, being about 100 for practical transistors: for a small variation in $j_{b}$ (input signal), there is a large variation in $j_{c}$ (output signal). In the following we show that the electron flow in MBTs brings about spin accumulation (nonequilibrium spin) in the collector, proving the possibility of the electrical spin injection. We also show that $\beta$ depends on both $\alpha_{e}$ and on $\alpha_{0 b}$, predicting a spin control of the gain.

Electrical spin injection. Our goal is to calculate how much spin polarization $\alpha_{c}=s_{c} / N_{d c}$ will accumulate in the collector in response to the nonequilibrium spin $\alpha_{e}$ and the equilibrium spin $\alpha_{0 b}$. Although, strictly speaking, $\alpha_{c}$ is evaluated at the point $c$ at the depletion layer edge (see Fig. 1), it also represents the spin accumulation within the spin diffusion length into the collector. We model the emitter-base junction as a forward biased magnetic $p-n$ junction with equilibrium spin polarization $\alpha_{0 b}$ in the $p$ region (base) and source spin polarization $\alpha_{c}$ in the $n$ region (emitter). Our theory of magnetic $p-n$ junctions ${ }^{7}$ determines the electron $n_{b e}$ and spin $s_{b e}$ densities at the be region (see Fig. 1) at the depletion layer edge:

$$
n_{b e}=n_{0 p} e^{q V_{b e} / k_{\mathrm{B}} T}\left(1+\alpha_{e} \alpha_{0 b}\right),
$$

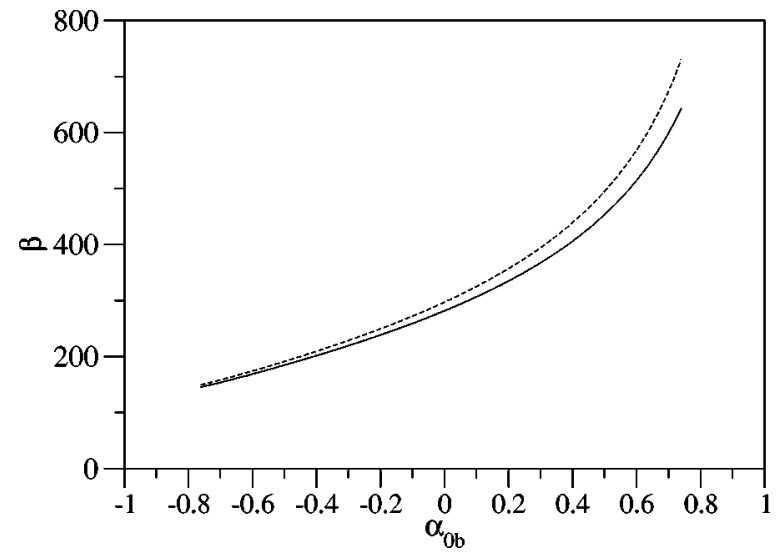

FIG. 2. Calculated current amplification coefficient $\beta$ as a function of $\alpha_{0 b}$ for a fixed $\alpha_{e}$. The dashed line is Eq. (10).

$$
s_{b e}=n_{0 b} e^{q V_{b e} / k_{\mathrm{B}} T}\left(\alpha_{0 b}+\alpha_{e}\right) .
$$

The nonequilibrium electron density injected into the base depends on the product $\alpha_{e} \alpha_{0 b}$, realizing the SilsbeeJohnson spin-charge coupling. ${ }^{29,30}$ If $\alpha_{e}=0$, Eq. (2) reduces to the standard Shockley's equation ${ }^{2}$ for the nonequilibrium minority electron density in a biased $p-n$ junction.

We next model the base-collector junction as another magnetic $p-n$ junction. This junction is reverse biased, and has both the equilibrium spin polarization $\alpha_{0 b}$ and the source spin density $s_{b e}$ in the $p$ region (base). This is the case of a magnetic solar cell, ${ }^{5}$ since the electron and the source spin densities in the $p$ region mimic the carrier and spin generation by light. For this case our theory ${ }^{7}$ gives

$$
s_{c} \approx \gamma_{1} s_{b e}=\gamma_{1} n_{0 b} e^{q V_{b e} / k_{\mathrm{B}} T}\left(\alpha_{0 b}+\alpha_{e}\right),
$$

where $\gamma_{1} \approx\left(L_{s} / w_{b}\right) \tanh \left(w_{c} / L_{s}\right)$. The accumulated spin polarization, which is the measure of the electrical spin injection efficiency, is $\alpha_{c}=s_{c} / N_{d c}$. Typically the spin diffusion length in the collector $L_{s} \gg w_{b}$, which means that $\alpha_{c}$ can be a considerable fraction (say, $10 \%$ ) of $\alpha_{e}$ or $\alpha_{0 b}$. What is interesting in Eq. (4) is the fact that $\alpha_{0 b}$ plays the same role as $\alpha_{e}$ in the spin injection: the equilibrium spin can cause spin accumulation in the low injection limit, because it leads first to nonequilibrium spin $s_{b e}$. This has no analog in magnetic diodes, where spin accumulation cannot result from the presence of just an equilibrium spin polarization.

Spin control of current amplification. When written in terms of $n_{b e}$, the formulas for the currents $j_{e}$ and $j_{c}$ are the same as for the standard (nonmagnetic) bipolar transistors derived by Shockley. ${ }^{2}$ After we write those formulas for the active forward regime, we substitute Eq. (2) for $n_{b e}$ and obtain the dependence of the currents (and of $\beta$ ) on $\alpha_{e}$ and $\alpha_{0 b}$.

The emitter current is

$$
j_{e}=j_{g b}^{n}\left(n_{b e} / n_{0 b}\right)+j_{g e}^{p}\left(p_{e} / p_{0 e}\right),
$$

where the electron generation current is $j_{g b}^{n}$ $=\left(q D_{n} / L_{n}\right) n_{0 b} \operatorname{coth}\left(w_{b} / L_{n}\right)$, the hole generation current is $j_{g e}^{p}=\left(q D_{p} / L_{p}\right) p_{0 e} \operatorname{coth}\left(w_{e} / L_{p}\right)$, and the injected hole density in the emitter is $p_{e}=p_{0 e} \exp \left(q V_{b e} / k_{\mathrm{B}} T\right)$. The collector current is comprised only of electrons (Fig. 1), 


$$
j_{c}=j_{g b}^{n}\left(n_{b e} / n_{0 b}\right) \cosh \left(w_{b} / L_{n}\right) .
$$

After evaluating $j_{b}=j_{e}-j_{c}$ and substituting Eq. (2) for $n_{b e}$, it is straightforward to show that in the narrow base limit $\left(w_{b} \ll L_{n}, L_{s}\right)$ the gain is

$$
\beta=1 /\left(\alpha_{T}^{\prime}+\gamma^{\prime}\right) \text {, }
$$

where we use the standard transistor notation, ${ }^{3}$

$$
\begin{aligned}
& \alpha_{T}^{\prime}=\left(w_{b} / L_{n}\right)^{2} / 2, \\
& \gamma^{\prime}=\frac{N_{b} D_{p}}{N_{e} D_{n}} \frac{w_{b}}{L_{p} \tanh \left(w_{e} / L_{p}\right)} \frac{\sqrt{1-\alpha_{0 b}^{2}}}{\left(1+\alpha_{e} \alpha_{0 b}\right)} .
\end{aligned}
$$

The factor $\alpha_{T}^{\prime}$ determines how much electrons will recombine in the base, thus not reaching the collector. This factor is not affected by the presence of spin, and is the same as in the standard transistors. The factor $\gamma^{\prime}$ is related to the emitter injection efficiency, since it measures the proportion of the electron flow in the emitter current (where both electrons and holes contribute). This factor does depend on the spin. To get the maximum amplification, both $\alpha_{T}^{\prime}$ and $\gamma^{\prime}$ need to be small. For the most efficient spin control of $\beta$, one needs $\alpha_{T}^{\prime} \lesssim \gamma^{\prime}$, the case of Si-based transistors which have slow carrier recombination. In this case,

$$
\beta\left(\alpha_{e}, \alpha_{0 b}\right)=\beta\left(\alpha_{e}=0, \alpha_{0 b}=0\right) \times \frac{1+\alpha_{e} \alpha_{0 b}}{\sqrt{1-\alpha_{0 b}^{2}}} .
$$

The current amplification is affected by both $\alpha_{e}$ and $\alpha_{0 b}$.

As an illustration we calculate $\beta$ as a function of $\alpha_{0 b}$ for a MBT with $\alpha_{e}=0.9$ and with generic material parameters, specified for a Si-like transistor (see Fig. 2). The nominal widths of the emitter, base, and collector are 2, 1.5, and 2 $\mu \mathrm{m}$, respectively. The dopings are $N_{e}=10^{17}, N_{b}=10^{16}$, and $N_{c}=10^{15} \mathrm{~cm}^{-3}$. Electron (hole) diffusivities at room temperature are taken to be $D_{n}=100$ and $D_{p}=10 \mathrm{~cm}^{2} / \mathrm{s}$. The bias voltages are $V_{b e}=0.5$ and $V_{b c}=0 \mathrm{~V}$. The intrinsic carrier density $n_{i}=10^{10} \mathrm{~cm}^{-3}$ and the dielectric constant (needed to calculate the effective widths $w$ ) is 12 . The carrier and spin diffusion lengths (note that $\mathrm{Si}$ has long recombination and spin relaxation times ${ }^{31}$ ) are taken to be $L_{n}$ $=30 \mu \mathrm{m}, L_{p}=L_{s}=10 \mu \mathrm{m}$. The calculated $\beta$ varies strongly with the spin, following closely the approximate $\beta$ given by Eq. (10). The amplification is largest (smallest) for the parallel (antiparallel) orientation of the source and equilibrium spins.

We conclude that spin can be injected through MBTs and that current amplification can be controlled by both the source and the equilibrium spin, making MBTs attractive for spintronic applications.

This work was supported by DARPA, the NSF-ECS, and the US ONR.

${ }^{1}$ A preliminary version of this paper was posted as J. Fabian and I. Žutić and S. Das Sarma, cond-mat/0211639, 2002.

${ }^{2}$ W. Shockley, Electrons and holes in semiconductors (Van Nostrand, Princeton, 1950).

${ }^{3}$ S. Tiwari, Compound Semiconductor Device Physics (Academic, San Diego, 1992)

${ }^{4}$ I. Zutić, J. Fabian, and S. Das Sarma, Phys. Rev. B 64, 121201 (2001).

${ }^{5}$ I. Žutić, J. Fabian, and S. Das Sarma, Appl. Phys. Lett. 79, 1558 (2001).

${ }^{6}$ I. Žutić, J. Fabian, and S. Das Sarma, Phys. Rev. Lett. 88, 066603 (2002).

${ }^{7}$ J. Fabian, I. Žutić, and S. Das Sarma, Phys. Rev. B 66, 165301 (2002).

${ }^{8}$ R. Fiederling, M. Kleim, G. Reuscher, W. Ossau, G. Schmidt, A. Waag, and L. W. Molenkamp, Nature (London) 402, 787 (1999).

${ }^{9}$ Y. Ohno, D. K. Young, B. Beschoten, F. Matsukura, H. Ohno, and D. D. Awschalom, Nature (London) 402, 790 (1999).

${ }^{10}$ B. T. Jonker, Y. D. Park, B. R. Bennett, H. D. Cheong, G. Kioseoglou, and A. Petrou, Phys. Rev. B 62, 8180 (2000).

${ }^{11}$ P. R. Hammar and M. Johnson, Appl. Phys. Lett. 79, 2591 (2001).

${ }^{12}$ X. Hao, J. S. Moodera, and R. Meservey, Phys. Rev. B 42, 8235 (1990).

${ }^{13}$ H. Ohno, H. Munekata, T. Penney, S. von Molnár, and L. L. Chang, Phys. Rev. Lett. 68, 2664 (1992).

${ }^{14} \mathrm{H}$. Ohno, Science 281, 951 (1998).

${ }^{15}$ H. Saito, V. Zayets, S. Yamagata, and K. Ando, Phys. Rev. Lett. 90, 207202 (2003).

${ }^{16}$ M. Kohda, Y. Ohno, K. Takamura, F. Matsukura, and H. Ohno, Jpn. J. Appl. Phys. 40, L1274 (2001).

${ }^{17}$ E. Johnston-Halperin, D. Lofgreen, R. K. Kawakami, D. K. Young, L. Coldren, A. C. Gossard, and D. D. Awschalom, Phys. Rev. B 65, 041306 (2002).

${ }^{18}$ N. Samarth, S. H. Chun, K. C. Ku, S. J. Potashnik, and P. Schiffer, Solid State Commun. 127, 173 (2003).

${ }^{19}$ M. Field (private communication, 2003).

${ }^{20}$ S. Datta and B. Das, Appl. Phys. Lett. 56, 665 (1990).

${ }^{21}$ D. J. Monsma, J. C. Lodder, T. J. A. Popma, and B. Dieny, Phys. Rev. Lett. 74, 5260 (1995).

${ }^{22}$ M. Johnson, Science 260, 320 (1993).

${ }^{23}$ C. Ciuti, J. P. McGuire, and L. J. Sham, Appl. Phys. Lett. 81, 4781 (2002).

${ }^{24}$ S. van Dijken, X. Jiang, and S. S. P. Parkin, Phys. Rev. Lett. 90, 197203 (20).

${ }^{25}$ X. Jiang, R. Wang, S. van Dijken, R. Shelby, R. Macfarlane, G. S. Solomon, J. Harris, and S. S. P. Parkin, Phys. Rev. Lett. 90, 256603 (2003).

${ }^{26}$ G. E. W. Bauer, A. Brataas, Y. Tserkovnyak, and B. J. van Wees, Appl. Phys. Lett. 82, 3928 (2003).

${ }^{27}$ T. Dietl, in Handbook of Semiconductors edited by T. S. Moss and S. Mahajan (North-Holland, New York, 1994), Vol. 3, p. 1279.

${ }^{28}$ F. Meier and B. P. Zakharchenya, in Optical Orientation (North-Holland New York, 1984).

${ }^{29}$ R. H. Silsbee, Bull. Magn. Reson. 2, 284 (1980).

${ }^{30}$ M. Johnson and R. H. Silsbee, Phys. Rev. Lett. 55, 1790 (1985).

${ }^{31}$ D. J. Lepine, Phys. Rev. B 2, 2429 (1970). 